\title{
Measurements of the average muon energy in inclined muon bundles in the NEVOD-DECOR experiment
}

\section{E.A. Yurina ${ }^{a}, *$ N.S. Barbashina ${ }^{a}$, A.G. Bogdanov $^{a}$, S.S. Khokhlov ${ }^{a}$, V.V. Kindin ${ }^{a}$, R.P. Kokoulin ${ }^{a}$, K.G. Kompaniets ${ }^{a}$, G. Mannocchi ${ }^{b}$, A.A. Petrukhin ${ }^{a}$,} V.V. Shutenko ${ }^{a}$, G. Trinchero $^{b}$, I.I. Yashin ${ }^{a}$

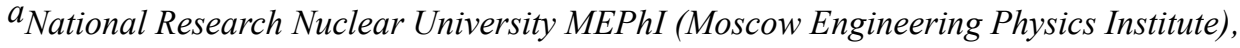
Kashirskoe shosse 31, Moscow, 115409, Russia

${ }^{b}$ Osservatorio Astrofisico di Torino - INAF,

Strada Osservatorio 20, Pino Torinese, 10025, Italy

E-mail: eayurina@mephi.ru

In the NEVOD-DECOR experiment, the study of the energy characteristics of muon bundles is carried out, aimed at the solution of "muon puzzle" (the excess of multi-muon events in cosmic rays in comparison with the results of calculations). The experimental complex includes a Cherenkov water calorimeter and a coordinate-tracking detector. The energy deposit of muon bundles is measured from the response of the NEVOD calorimeter, and coordinate-tracking detector DECOR allows to determine the number of muons in the bundles and their arrival directions. Experimental estimates of the average muon energy in the bundles and their dependence on the zenith angle and primary energy in the range from $10 \mathrm{PeV}$ to $1000 \mathrm{PeV}$ have been obtained and compared with the results of calculations performed using the simulation based on the CORSIKA software package using models of hadronic interactions QGSJET-II-04 and SIBYLL-2.3c. 


\section{Introduction}

Investigations of cosmic rays with energies of the order of $\mathrm{PeV}$ and higher are based on indirect measurements of various components of extensive air showers (EAS). Therefore, for obtaining of the characteristics of the primary cosmic ray (PCR) flux, adequate physical models of EAS are required. The observation of an excess of muons in the EAS relative to predictions in the region of PCR energies above $10^{17} \mathrm{eV}$ was reported by the authors of many experiments. In particular, in the NEVOD-DECOR experiment $[1,2,3]$ the dependence of the muon excess on the PCR energy was measured. In this experiment, EAS investigations are based on the method of local muon density spectra (LMDS) [1] which allows in the frames of one experiment to study EAS muon bundles in a wide range of PCR energies from 10 to $1000 \mathrm{PeV}$ and higher. Measurements at the Pierre Auger Observatory $[4,5]$ also confirmed an excessive number of muons in inclined EAS in comparison with calculations in the energy range about $10^{19} \mathrm{eV}$.

None of the modern models of hadron interactions, tuned with the available data from the Large Hadron Collider (LHC), can quantitatively describe the production of muons for primary energies above $10^{17} \mathrm{eV}$. The excess of multi-muon events growing with the PCR energy is called the "muon puzzle" [6]. The results of investigations of various experiments on the "muon puzzle" were brought together by the International Working Group on Hadronic Interactions and Shower Physics (WHISP) [7, 8].

The key to the muon excess solution can be the study of the energy characteristics of the EAS muon component and their changes with increasing energy of primary particles. A possible approach to such studies is to measure the energy deposit of cosmic ray muon bundles in the detector matter. Since the average energy loss of muons linearly depends on their energy, the deviation of the experimental dependence from the expected one will indicate the appearance of high-energy muons, which can be formed as a result of a possible inclusion of new physical processes. Also, the study of the energy characteristics of muon bundles and their comparison with the results of calculations allows to check the existing models of hadronic interactions.

The only setup in the world that currently measures the energy deposit of muon bundles is the NEVOD-DECOR experiment, which includes a Cherenkov water calorimeter (CWC) NEVOD with a volume of $2000 \mathrm{~m}^{3}$ and a coordinate-tracking detector DECOR with an area of about $70 \mathrm{~m}^{2}$. Previous research results on the study of the specific energy deposit of the bundles were presented in [9], and the first results on the average energy of muons in the bundles were published in [10]. In this article, additional statistics of muon bundles has been included and some refinements in the analysis procedure have been introduced.

\section{Experimental setup and data}

The measuring system of the CWC NEVOD $[11,12]$ is a space-centered lattice of 91 quasispherical modules (QSM) which can be represented in the form of 7 planes in the direction of each of the axes of a rectangular coordinate system. Adjacent planes consist of 16 or 9 QSMs combined in the form of strings (4 or 3 QSMs in a string respectively). Each QSM includes 6 FEU-200 PMT with flat $15 \mathrm{~cm}$ diameter photocathodes directed along the axes of the orthogonal coordinate system. The dynamic range of the recording electronics is from 1 to $10^{5}$ photoelectrons (ph.e.) for each PMT due to the use of two-dinode signal pickup. It allows to carry out calorimetric studies, in particular, to measure the energy deposit of muon bundles. 
The coordinate-tracking detector DECOR [13] consists of 8 supermodules (SMs) located in the galleries of the experimental building on three sides of the NEVOD water volume. The effective area of one SM is $8.4 \mathrm{~m}^{2}$. Each SM represents 8 vertical planes of plastic streamer tubes with a resistive cathode coating and a two-coordinate system for reading signals from external strips: $256 \mathrm{X}$ - and $256 \mathrm{Y}$-channels in each plane. The angular and spatial accuracy of the reconstruction of muon tracks crossing the SM is better than 1 degree and $1 \mathrm{~cm}$ respectively.

The technique for selection of muon bundles in a coordinate detector is based on the parallelism of particle tracks recorded by the setup. The procedure for selection of events according to the DECOR data consists of several stages: trigger level (3-fold coincidences of signals from different SMs within a time gate of $250 \mathrm{~ns}$ ); program selection and reconstruction of candidate events containing quasi-parallel tracks (within a 5-degree cone); final classification of events and counting of tracks by operators.

The registered tracks of muon bundle event in DECOR SMs are shown in fig. 1. Reconstructed muon bundle event recorded jointly by the NEVOD and DECOR setups is shown in fig. 2.

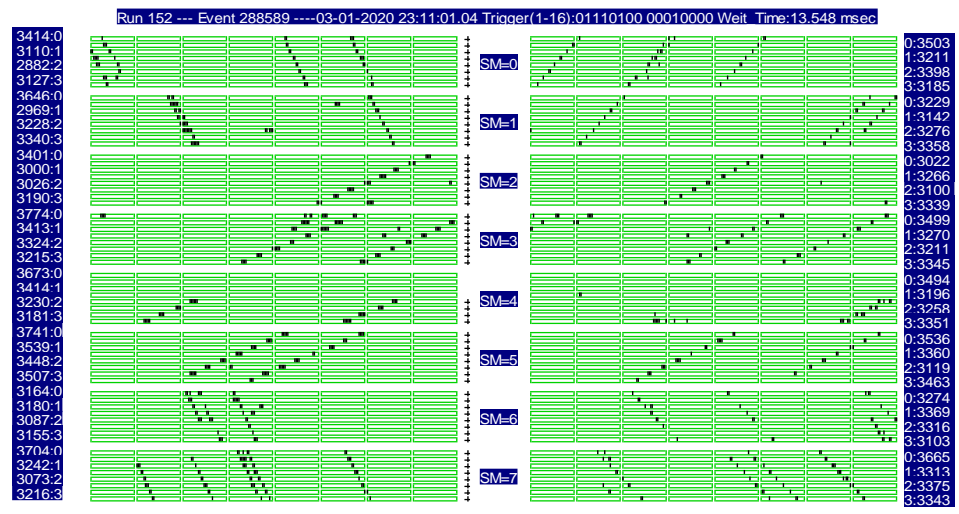

Figure 1: An example of an event with a muon bundle registered by supermodules of the DECOR detector. Right $-X$-projection, projected zenith angle; left $-Y$-projection, azimuth angle.

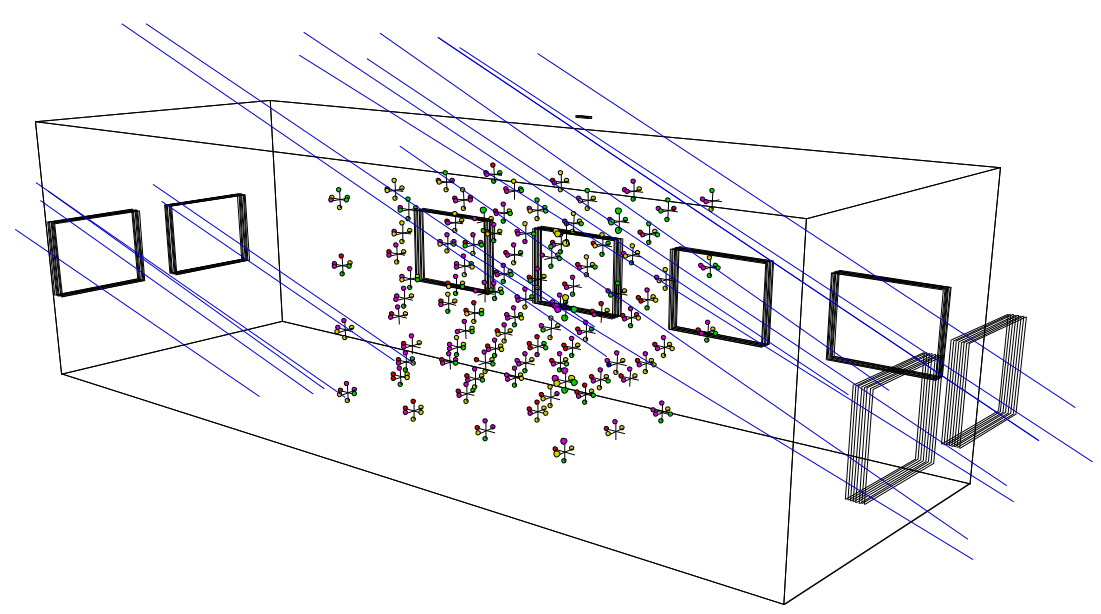

Figure 2: Muon bundle registered jointly by the NEVOD and DECOR detectors. The lines show reconstructed muon tracks according to DECOR data; small circles are hit PMTs in CWC NEVOD; large rectangles are DECOR supermodules. 
Events with zenith angles greater than $55^{\circ}$ were selected, where practically only pure muon component of the EAS reaches the Earth's surface. At smaller zenith angles muon bundles can be accompanied by electron-photon and hadron components of the EAS, which complicate the geometric reconstruction of the tracks. Events were selected in two 60-degree sectors in azimuth angle, where most part of the coordinate detector (six of eight SMs) is shielded by the water volume of the NEVOD detector. Over a long observation period (from July 2013 to March 2021, 53 thousand hours of "live" time) 90,165 events with bundles with a multiplicity (number of muons in a bundle) $m \geq 5$ and a zenith angle $\theta \geq 55^{\circ}$ were selected.

Since the dimensions of the detector are much smaller than the characteristic dimensions of the muon component of the EAS on the Earth's surface, in the first approximation the detector can be considered as a point-like one. Then, in an individual event, the local muon density $D$ is estimated as the ratio of multiplicity $m$ to the effective area of the installation in a given direction $S_{\text {det }}$, taking into account the bias of the estimate due to Poisson fluctuations in the number of particles entering the detector and the steeply falling density spectrum [1]:

$$
D=(m-\beta) / S_{\text {det }},
$$

where $\beta \sim 2.1$ is the integral LMDS slope [1].

As a measure of the energy deposit of muon bundles in the calorimeter, the sum $\Sigma$ of the signals of all triggered PMTs of the NEVOD detector (in ph.e. units) is used. In the first approximation, the total energy deposit is proportional to the muon density $D$ in the event; therefore, in what follows, the specific energy deposit $\Sigma / D$ as the number of photoelectrons divided by the estimate of the muon density obtained from the coordinate-tracking detector data was considered.

\section{Simulation of the NEVOD-DECOR response to muon bundles}

The energy deposits of muon bundles with fixed muon energies of $100 \mathrm{GeV}$ were simulated for the NEVOD-DECOR setups. Events with bundles were simulated according to the local muon density spectrum with a slope close to the experimental one. The simulation takes into account the physical features of the setups and the conditions of hardware, software and operator selection of events with muon bundles. For events satisfying the selection conditions, the response of the Cherenkov water calorimeter NEVOD was calculated using the Geant4 package $[14,15]$.

The model of the CWC NEVOD was verified by comparing the results of calculating of the response of the NEVOD detector to the passage of single near-horizontal muons with experimental data. In this case, the coordinates of the entrance to the water tank and the direction of motion of single muons were taken from the experimental data. Near-horizontal muons were selected using supermodules of the detector DECOR located in opposite short galleries of the experimental complex building NEVOD (fig. 3). In the simulation, the muon energy was sampled close to the experimental conditions with a distribution over the single muon energy spectrum and, on average, was equal to $100 \mathrm{GeV}$. 


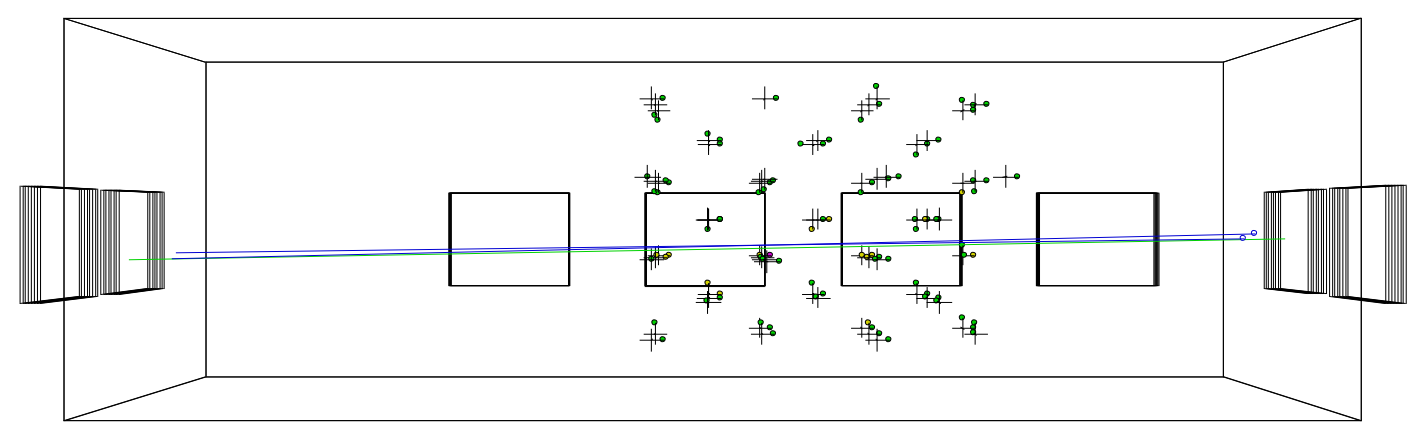

Figure 3: An example of an event with a near-horizontal muon (passage of a particle through DECOR SMs located in opposite short galleries).

The accuracy of the local muon density $D$ estimation was improved on the basis of the simulation of the DECOR SMs response to the passage of muon bundles using the Monte Carlo method. In the simulation three significant effects were taken into account: the condition for triggering a supermodule (triggering of at least two even and two odd planes in the $Y$-projection ), selection by the reconstruction program and operators of muon tracks with at least 5 triggered planes in the $X$-projection, as well as the inefficiency of registration associated with the passage of tracks through the gaps between the chambers, their walls, through the chambers that were turned off due to malfunctions. As a result, the correction was obtained as the ratio of the local density based on simulation to the local density from the results of the analytical calculation $S_{\text {det. }}$ This correction also includes the track masking effect (one or more tracks are masked in both projections), which is significant at muon density of more than 2 particles per $\mathrm{m}^{2}$.

\section{Results}

The residual contribution from the electron-photon and hadron components of the EAS decreases exponentially with $\sec \theta$ but remains appreciable in the overlapping range of zenith angles $\left(55^{\circ}-65^{\circ}\right)$ in the dependence of the average specific energy deposit on the zenith angle. In fig. 4, the dependences of the average specific energy deposit on the zenith angle are shown (black circles are experimental specific energy deposit, red squares are specific energy deposit of the expected muon contribution). The expected muon contribution was subtracted from the experimental dependence. From the results of fitting of the obtained curve (after this subtracting) by exponential function we estimated the values of contribution from the electromagnetic and hadron EAS components at zenith angles of interest $\theta=57.5^{\circ}$ and $\theta=62.5^{\circ}$, which were $(4.6 \pm 1.0) \%$ and $(0.7 \pm 0.3) \%$ respectively. When obtaining the experimental dependence of the average specific energy deposit, this contribution was taken into account. 


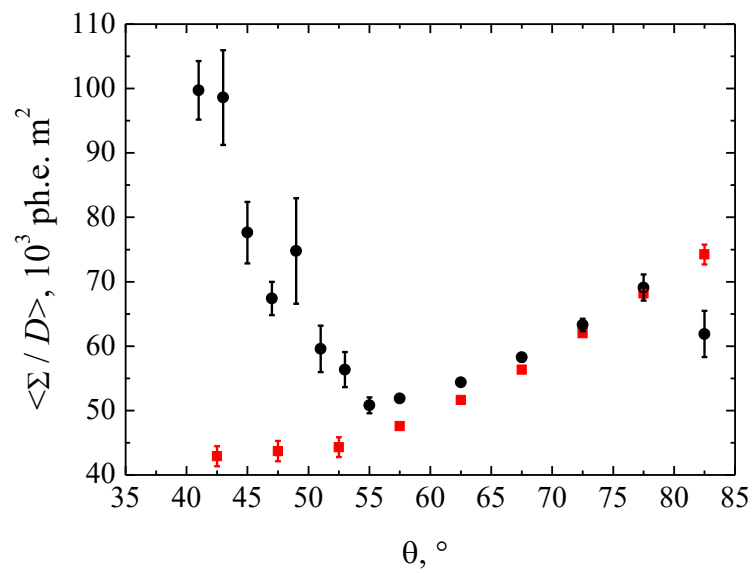

Figure 4: Dependences of experimental (black circles) and expected specific energy deposit (expected muon contribution, red squares) on zenith angle.

In fig. 5 the experimental and calculated dependences of the average specific energy deposit on the zenith angle are given. Black circles are experimental data, red squares are simulation results (for a fixed muon energy of $100 \mathrm{GeV}$ ). As can be seen from the figure, there is an increase in the average specific energy deposit with an increase in the zenith angle (both for the experiment and for the simulation results). However, the experimental points are significantly higher than the simulation, which is due to the contribution of various energies of muons in the bundles, and the average energy of muons in the bundles is higher than $100 \mathrm{GeV}$.

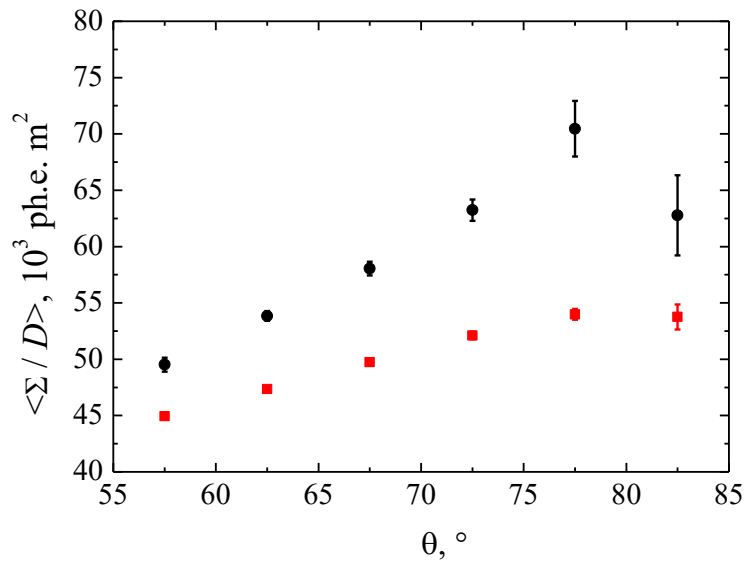

Figure 5: Dependence of the average specific energy deposit of muon bundles on the zenith angle: the experiment (black circles) and simulation results (red squares for a fixed muon energy of 100 $\mathrm{GeV})$.

Using the dependence of the average specific energy loss in water on the muon energy according to tabulated data [16] normalized to loss at $100 \mathrm{GeV}$, which is almost linear in the range of hundreds of $\mathrm{GeV}$, from the ratio of the measured and simulated specific energy deposit we can obtain the dependence of the average energy of muons in the bundles on the zenith angle in the NEVOD-DECOR experiment. In fig. 6a the obtained dependence of the average energy of muons in the bundles on the zenith angle is shown. The arrows indicate the calculated mean logarithmic energies of primary particles [1]. The curves were obtained by simulation of the muon component of EAS formed by primary protons (bottom pair of curves) and iron nuclei (top pair of curves) in 
the CORSIKA software package [17] for models of hadronic interactions QGSJET-II-04 [18] (solid lines) and SIBYLL-2.3c [19] (dashed lines).

The average energy of muons in the bundles (for a given primary particle, its energy $E_{0}$ and direction, as well as the model of hadron interactions) was calculated using the LMDS method [1] as:

$$
\left\langle E_{\mu}\right\rangle=\frac{\int\left\langle E_{\mu}(\mathbf{r})\right\rangle\left[\rho\left(E_{0}, \mathbf{r}\right)\right]^{\beta} d S}{\int\left[\rho\left(E_{0}, \mathbf{r}\right)\right]^{\beta} d S},
$$

where $\beta=2.2$ is a fixed slope of the LMDS; $\mathbf{r}$ is the point in the cross section of the shower; $\rho\left(E_{0}, \mathbf{r}\right)$ is the average lateral distribution function (LDF) of muons (density of particles in point $\mathbf{r}$ ). Interpolation of the results of these calculations allows to obtain the expected average energies of muons in the bundles for given zenith angles and local densities.

The fig. $6 a$ shows the increase in the average energy of muons in the bundles with an increase in the zenith angle, the experimental results are in a good agreement with the expectation.

In fig. $6 b$ the dependence of the average muon energy on the local muon density obtained for the zenith angle interval $65^{\circ}-75^{\circ}$ is presented. The designations of the curves and arrows are the same as in fig. $6 a$. The curves in the figure were obtained by simulation of showers for protons and iron nuclei of the PCR for a fixed zenith angle $\theta=69^{\circ}$. Experimental data indicate an increase in the average energy of muons in the bundles in comparison with the calculated results at large muon densities corresponding to PCR energies above $10^{17} \mathrm{eV}$.
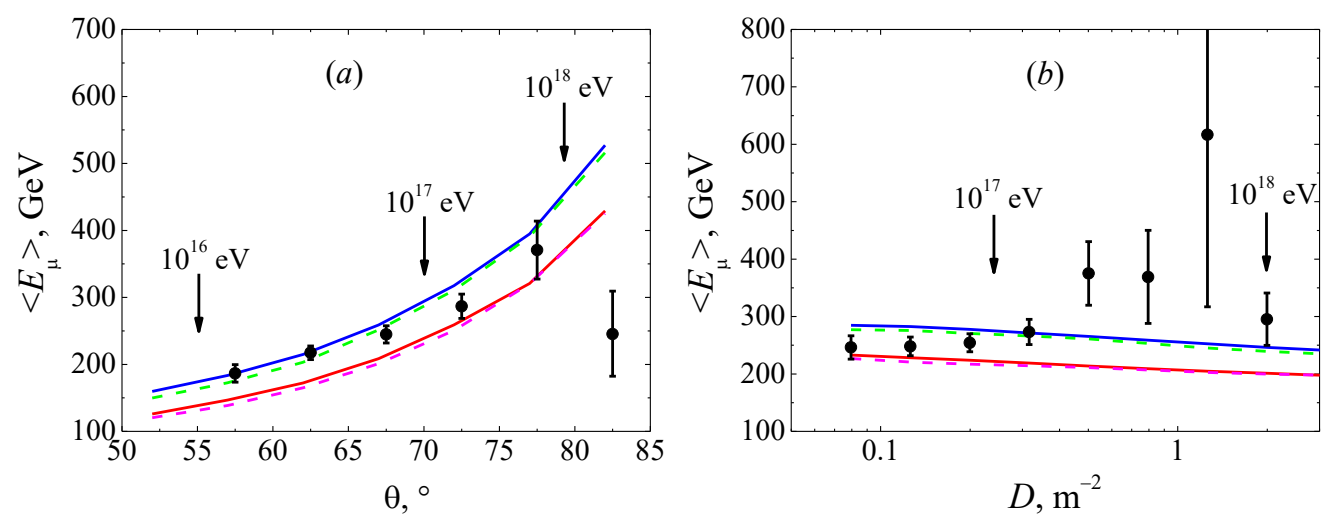

Figure 6: Dependence of the average energy of muons in the bundles on the zenith angle $(a)$ and on the local muon density for the interval of zenith angles $\theta=65^{\circ}-75^{\circ}(b)$. See the text for explanation of designations.

\section{Conclusion}

In the NEVOD-DECOR experiment, estimates of the average energy of muons in the bundles were obtained at different zenith angles and local densities, which correspond to the energies of primary particles of $10^{16}-10^{18} \mathrm{eV}$. The obtained data are compared with the calculation results based on EAS simulation in the CORSIKA software package. For large muon densities, corresponding to primary energies greater than $10^{17} \mathrm{eV}$, an increase in the average energy of muons in the bundles in comparison with the calculation results is observed. 


\section{Acknowledgments}

The work has been performed at the Unique Scientific Facility "Experimental complex NEVOD" with the support of the Ministry of Science and Higher Education of the Russian Federation, Project "Fundamental problems of cosmic rays and dark matter", No 0723-2020-0040. The simulation was carried out using the resources of the high-performance computing center of MEPhI.

\section{References}

[1] A.G. Bogdanov et al., Phys. At. Nucl. 73, 1852 (2010).

[2] A.G. Bogdanov et al., Astropart. Phys. 98, 13 (2018).

[3] A.G. Bogdanov et al., J. Phys.: Conf. Ser. 1690, 012007 (2020).

[4] L. Nellen (Pierre Auger Collab.), J. Phys.: Conf. Ser. 409, 012107 (2013).

[5] A. Aab et al. (Pierre Auger Collab.), Phys. Rev. D 91, 032003 (2015).

[6] A.A. Petrukhin, Nucl. Instrum. Methods Phys. Res., Sect. A 742, 228 (2014).

[7] H.P. Dembinski et al. (EAS-MSU, IceCube, KASCADE-Grande, NEVOD-DECOR, Pierre Auger, SUGAR, Telescope Array, and Yakutsk EAS Array Collabs.), EPJ Web Conf. 210, 02004 (2019).

[8] L. Cazon (EAS-MSU, IceCube, KASCADE-Grande, NEVOD-DECOR, Pierre Auger, SUGAR, Telescope Array, and Yakutsk EAS Array Collabs.), PoS (ICRC2019), 214 (2019).

[9] E.A. Yurina et al., Phys. At. Nucl. 82, 680 (2019).

[10] E.A. Yurina et al., Bull. Russ. Acad. Sci. Phys. 85, 455 (2021).

[11] A.A. Petrukhin, Phys. Usp. 58, 486 (2015).

[12] V.V. Kindin et al., Instrum. Exp. Tech. 61, 649 (2018).

[13] N.S. Barbashina et al., Instrum. Exp. Tech. 43, 743 (2000).

[14] S. Agostinelli et al., Nucl. Instrum. Methods Phys. Res., Sect. A 506, 250 (2003).

[15] J. Allison et al., Nucl. Instrum. Methods Phys. Res., Sect. A 835, 186 (2016).

[16] D.E. Groom et al., At. Data Nucl. Data Tables 78, 183 (2001).

[17] D. Heck et al., Tech. Rep. FZKA-6019, Forschungszentrum Karlsruhe (1998).

[18] S. Ostapchenko, Phys. Rev. D 89, 074009 (2014).

[19] F. Riehn et al., PoS (ICRC2017), 301 (2018). 